\title{
Removal of malformation in cerebral proliferative angiopathy: illustrative case
}

\author{
Gwang Yoon Choi, MD, Hyuk Jai Choi, MD, PhD, Jin Pyeong Jeon, MD, PhD, Jin Seo Yang, MD, PhD, Suk-Hyung Kang, MD, PhD, and \\ Yong-Jun Cho, MD, PhD
}

Department of Neurosurgery, Hallym University Chuncheon Sacred Heart Hospital, Chuncheon, Gangwon, Republic of Korea

\begin{abstract}
BACKGROUND Cerebral proliferative angiopathy (CPA) is a rare vascular disorder distinct from arteriovenous malformation. Because of the disorder's rarity, there is still a controversy on the most promising treatment method for CPA. However, several meta-analysis articles suggest indirect vascularization such as encephalo-duro-arterio-synangiosis as an effective way of treating symptoms that are medically uncontrolled.
\end{abstract}

OBSERVATIONS The authors describe a case of an 11-year-old boy with this disease, who had epilepsy that was intractable despite conservative management. The patient recovered from his symptoms after the vascular malformation was surgically removed. This is the first reported case of surgical removal in CPA.

LESSONS Although further investigation on the best treatment for CPA is needed, the authors believe surgical intervention may also be an effective treatment modality when a patient presents with persisting symptoms.

https://thejns.org/doi/abs/10.3171/CASE2095

KEYWORDS cerebral proliferative angiopathy; surgical treatment; indirect vascularization

Cerebral proliferative angiopathy (CPA) is a rare vascular disorder that is defined angiographically as a diffuse vascular network forming a poorly defined nidus, which is supplied by multiple similar-sized feeding vessels and transdural channels. Interspersed normal brain parenchyma differentiates CPA from classic arteriovenous malformations (AVMs). ${ }^{1}$ Resection of the abnormal vessels can be challenging owing to the possibility of damaging the healthy tissues within. This case describes successful surgical removal of a CPA malformation in an 11year-old patient who presented with seizures. This treatment modality has not previously been attempted. ${ }^{2}$

\section{Illustrative Case}

\section{History and Presentation}

An 11-year-old boy presented with repeated seizures. The symptoms started 6 months earlier with an average of one episode a day accompanied by a brief loss of consciousness and aphasia. He recovered after approximately a minute, showing no postictal confusion.

The patient had mild right-sided motor weakness and trouble standing and maintaining balance at the age of 1 year. His parents took him to a local hospital and found out he had a poorly demarcated mass and a high level of vascularity in his left basal ganglia. Normal tissues appeared to be mingled within the lesion (Fig. 1A). The physician suggested a malignancy. Owing to the high risk of death involved in performing a biopsy, the parents decided to follow up routinely with brain imaging.

The patient's last follow-up was in 2008 , when he was 4 years old. Since then, he had not suffered from any other neurological symptoms. Magnetic resonance (MR) imaging showed no change in tumor size, but the signal void around the tumor became more apparent, suggesting increased vascularity (Fig. 1B). The physician continued to assume a diagnosis of malignancy and predicted a poor prognosis because of the aggressive nature of the disease. The patient had no further follow-up until he became symptomatic and visited our clinic.

Imaging revealed that the size of the lesion had increased from $4 \times$ $4.4 \mathrm{~cm}$ to $6 \times 5 \mathrm{~cm}$ during the 6-year interval, and edema around the lesion was aggravated. The tumor was a vascular structure resembling an AVM. MR angiography supported this suggestion by showing abnormal vessels from the left middle cerebral artery (MCA) and the lenticulostriate artery (LSA) (Fig. 2). However, transfemoral cerebral

ABBREVIATIONS AVM = arteriovenous malformation; $\mathrm{CPA}=$ cerebral proliferative angiopathy; $\mathrm{LSA}=$ lenticulostriate artery; $\mathrm{MCA}=$ middle cerebral artery; $\mathrm{MR}=$ magnetic resonance; TFCAG = transfemoral cerebral angiography.

INCLUDE WHEN CITING Published March 1, 2021; DOI: 10.3171/CASE2095.

SUBMITTED November 3, 2020. ACCEPTED January 11, 2021.

(C) 2021 The authors, CC BY-NC-ND 4.0 (http://creativecommons.org/licenses/by-nc-nd/4.0/). 

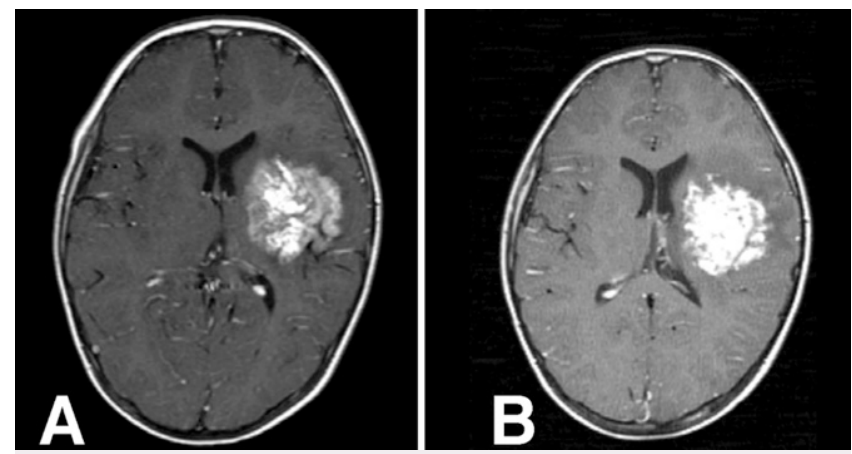

FIG. 1. Imaging over the course of the disease. A: Postcontrast T1 image at age 1 year. In the patient's left basal ganglia, a high level of vascularity and normal tissues appearing mixed in the lesion is observed. B: Postcontrast T1 image at age 4 years. The level of vascularity and the size of the lesion are increased.

angiography (TFCAG) confirmed the diagnosis of CPA with a large nidus supplied by multiple arteries stemming from the left MCA and LSA. No dominant feeder could be identified (Fig. 3).

\section{Operation and Postoperative Course}

The patient's parents initially wanted to continue conservative management. We administered valproate and topiramate for seizure control. However, the symptoms persisted over 3 months. Further increasing the dosage of medication was considered to be ineffective, and the patient and his parents agreed to surgery after a
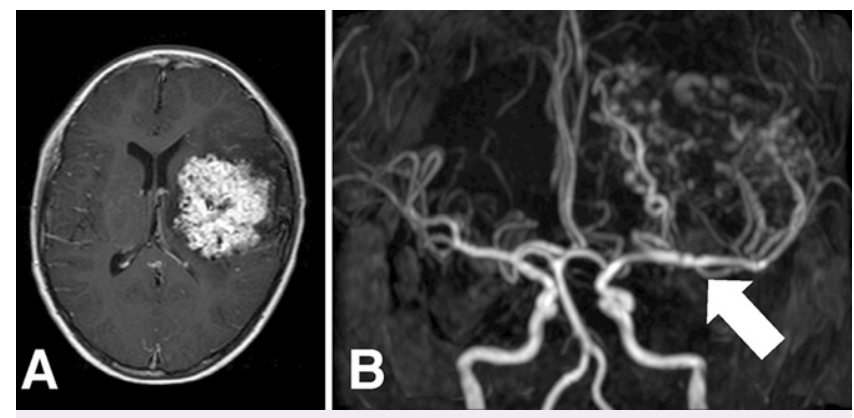

FIG. 2. Postcontrast T1 imaging (A) and MR angiography (B) on presentation. Nine years after the patient's first MR imaging, the level of vascularity and the size of the lesion were further increased. Feeders appear to arise from the branches of MCA and LSA (arrow).

full discussion of the possible complications, such as development of a new neurological deficit, intracranial hemorrhages, etc.

Microscopic operation was performed after left fronto-temporoparietal craniectomy. A relatively large feeder from the LSA was first occluded using an aneurysm clip, and the rest of the feeders were blocked, causing remarkable shrinkage of the nidus. The malformation was then resected, and the patient had no perioperative complication. Postoperative MR imaging and TFCAG showed nearcomplete elimination of the malformation (Figs. 4 and 5).

The patient continued antiepileptic medication at the same dose as in the preoperative period and has remained asymptomatic since the surgery. Also, he experienced no worsening of his hemiparesis. Annual

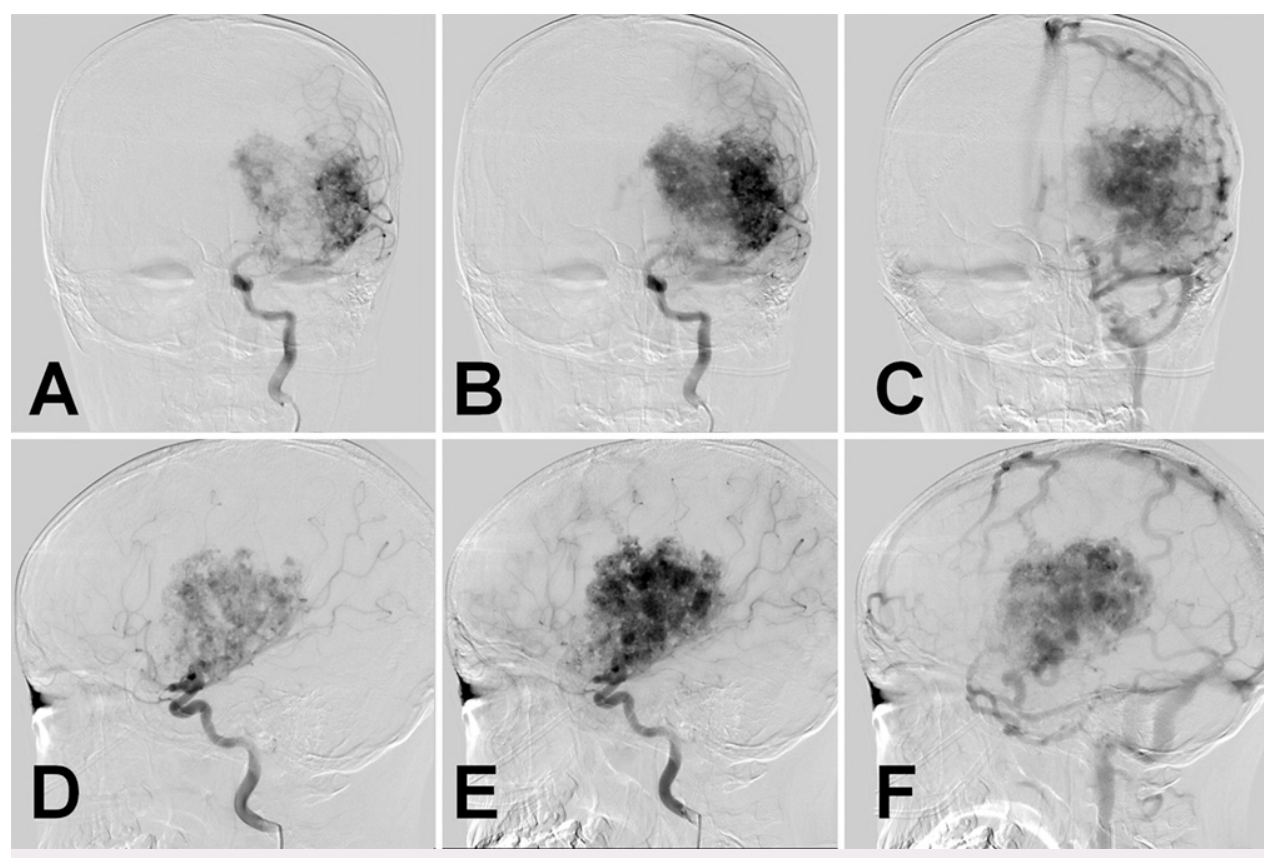

FIG. 3. TFCAG on admission: arterial phase ( $A$ and $\mathbf{D})$, capillary phase ( $B$ and $\mathbf{E})$, and venous phase ( $C$ and F). Although there were few normal tissues detectable within the malformation on MR imaging, TFCAG confirms the diagnosis of CPA. The lack of a dominant feeder, the transdural venous drainage, and the large size of the nidus are visible. 

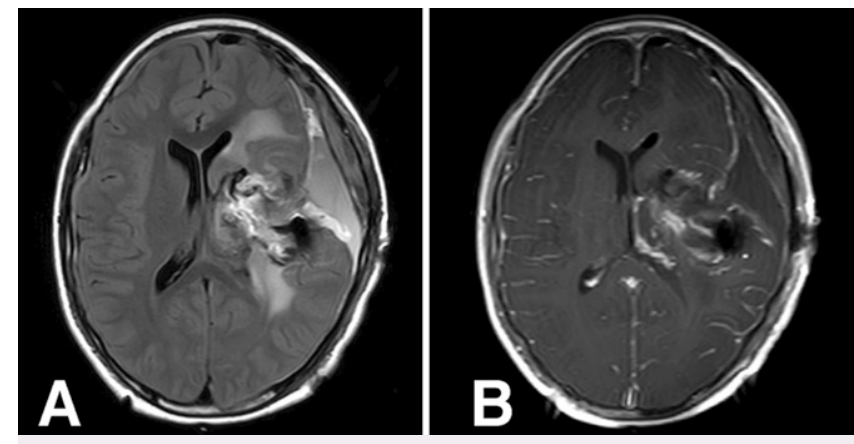

FIG. 4. Postoperative T2-weighted fluid-attenuated inversion recovery (A) and postcontrast T1-weighted (B) images show near-complete obliteration of the malformation.

follow-up TFCAGs have revealed no recurrence of the disease for 3 years.

\section{Discussion}

CPA is a rare disease entity separate from classic AVMs. It is angiographically characterized as having multiple small feeding arteries and draining veins with a transdural supply, but none of the feeders can be identified as dominant. Unlike AVMs, normal brain tissue is interspersed within the vascular abnormality. Patients usually present with seizures, headache, and stroke-like symptoms such as transient ischemic attacks. ${ }^{1}$

The exact pathophysiology is still unknown. However, it is hypothesized that there are chronic cortical ischemia and perinidal oligemia that elicit the proliferation of abnormal blood vessels. Also, it is currently considered that such ischemia and perinidal oligemia work as the main causes of the symptoms, and this theory works as the rationale for revascularization treatment. ${ }^{1,2}$

Many CPA treatment modalities have been attempted, including embolization, radiosurgery, medication, and revascularization., ${ }^{2,3}$ Revascularization such as encephalo-duro-arterio-synangiosis is currently considered a reasonable treatment option. ${ }^{2}$ Although the evidence is still lacking, revascularization seems to be relatively superior to other methods, and this finding is consistent with the suggested pathogenesis of the disease. Until now, there have been no reports on any type of surgical treatment or symptom improvement after total resection. ${ }^{2,3}$

\section{Observations}

In this case, when planning the optimal treatment method, we considered embolization of the structure improper because there were numerous feeding arteries with no dominant one, and it would be impossible to block every one of them. Furthermore, the patient had an anaphylactic reaction during TFCAG, possibly because of the contrast agent; hence, embolization was not possible. Radiosurgery also seemed inappropriate considering the current acute exacerbation of the symptoms. The patient had already tried antiepileptic medications, but his symptoms were consistent. Because there were few reports on revascularization for CPA at the time, we did not consider this as an option. Also, based on MR findings, neuronal tissues within and surrounding the lesion already showed considerable changes such as edema and gliosis, so we thought that marginal damage around the malformation would not result in significant neurological deficit. Therefore, although the risk was still substantial, we decided to remove the lesion completely.

We believe this case is the first report on the efficacy and prognosis of resection..$^{2,3}$ Current studies on the treatment of CPA have targeted ways to restore the blood supply of hypoperfused areas while leaving

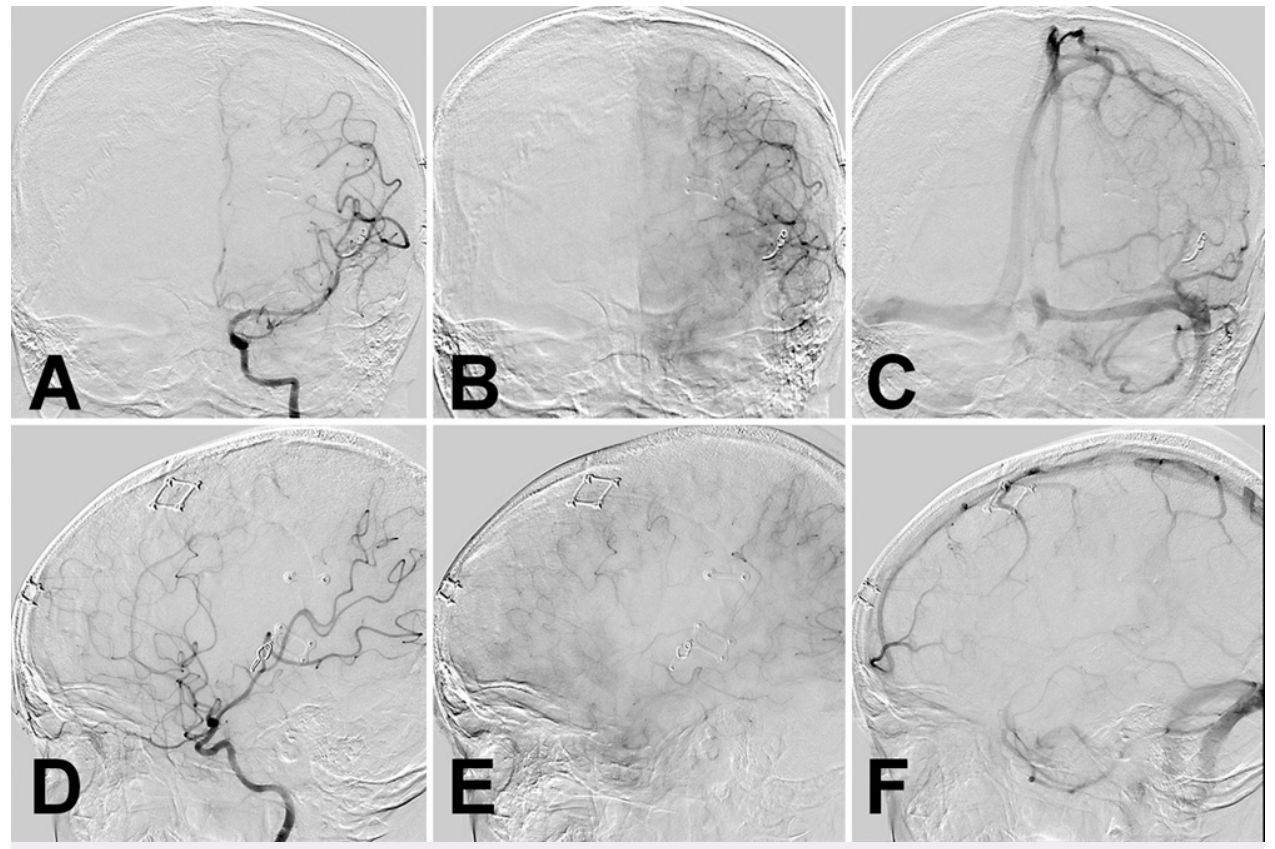

FIG. 5. Postoperative angiograms confirm removal of the lesion: arterial phase (A and $\mathbf{D})$, capillary phase ( $B$ and $\mathbf{E})$, and venous phase ( $\mathbf{C}$ and $\mathbf{F}$ ). 
the normal brain tissues within the lesion untouched. In this case, the treatment resulted in complete obliteration of the abnormal vessels, as well as the territory of normal parenchyma, and showed clinical improvement as control of the seizures.

\section{Lessons}

The reason for the improved symptoms without any new neurological change in this case could not be explained completely. Our first hypothesis is that, as perinidal oligemia is a possible cause of the symptoms, chronically hypoperfused perinidal tissues might be epileptogenic, and removing them might have led to the resolution of seizures. Of course, in our case, there was profound edema surrounding the perinidal region, probably resulting from persistent oligemia and mass effect, and this might have affected the postoperative result. Also, the normal brain parenchyma within the lesion might be neurologically inactive or even epileptogenic, despite its histological intactness, as shown in a previous study. ${ }^{1}$ In our case, the abnormal vessels were located in the basal ganglia, so removing the normal tissues could have led to some extent of neurological deficit, especially motor symptoms. However, the patient experienced no worsening of motor ability, raising suspicion of the tissue's actual contribution to his neurological function.

The success in this case demonstrates the need for investigating the efficacy of resection in the CPA population to control the symptoms. We hope further studies lead to a better understanding of this type of disease and the optimal treatment.

\section{References}

1. Lasjaunias PL, Landrieu P, Rodesch G, et al. Cerebral proliferative angiopathy: clinical and angiographic description of an entity different from cerebral AVMs. Stroke. 2008;39(3):878-885.

2. Somji M, McEachern J, Silvaggio J. Cerebral revascularization in cerebral proliferative angiopathy: a systematic review. Neurosurg Focus. 2019;46(2):E11.

3. Liu P, Lv X, Lv M, et al. Cerebral proliferative angiopathy: clinical, angiographic features and literature review. Interv Neuroradiol. 2016;22(1):101-107.

\section{Disclosures}

The authors report no conflict of interest concerning the materials or methods used in this study or the findings specified in this paper.

\section{Author Contributions}

Conception and design: HJ Choi, GY Choi, Yang. Acquisition of data: HJ Choi, GY Choi. Analysis and interpretation of data: HJ Choi, GY Choi. Drafting the article: GY Choi, Cho. Critically revising the article: HJ Choi, GY Choi. Reviewed submitted version of manuscript: HJ Choi, GY Choi, Jeon, Kang. Approved the final version of the manuscript on behalf of all authors: HJ Choi. Administrative/technical/material support: HJ Choi. Study supervision: Yang.

\section{Correspondence}

Hyuk Jai Choi: Hallym University Chuncheon Sacred Heart Hospital, Chuncheon, Gangwon, Republic of Korea. painsurgery@gmail.com. 